\begin{tabular}{|c|l|}
\hline Title & Singular solutions of first order differential equations \\
\hline Author(s) & Izumiya, Shyuichi \\
\hline Citation & Hokkaido University Preprint Series in Mathematics, 136, 2-6 \\
\hline Issue Date & 1992-02 \\
\hline DOI & 10.14943/83281 \\
\hline Doc URL & http://hdl.handle.net/2115/68883 \\
\hline Type & bulletin (article) \\
\hline File Information & pre136.pdf \\
\hline
\end{tabular}

Instructions for use 


\section{SINGULAR SOLUTIONS OF FIRST}

ORDER DIFFERENTIAL EQUATIONS

Shyuichi Izumiya

Series $\sharp 136$. February 1992 


\section{HOKKAIDO UNIVERSITY \\ PREPRINT SERIES IN MATHEMATICS}

$\sharp 111$ : T. Nishimori, A qualitative theory of similarity pseudogroups and an analogy of Sacksteder's theorem, 13 pages. 1991.

$\# 112$ : K. Matsuda, An analogy of the theorem of Hector and Duminy, 10 pages. 1991.

\#113: S. Takahashi, On a regularity criterion uo to the boundary for weak solutions of the Navier-Stokes equations, 23 pages. 1991.

$\sharp 114$ : T. Nakazi, Sum of two inner functions and exposed points in $H^{1}, 18$ pages. 1991.

\#115: A. Arai, De Rham operators, Laplacians, and Dirac operators on topological vector spaces, 27 pages. 1991.

$\sharp 116$ : T. Nishimori, A note on the classification of non-singular flows with transverse similarity structures, 17 pages. 1991.

$\sharp 117$ : T. Hibi, A lower bound theorem for Ehrhart polynomials of convex polytopes, 6 pages. 1991.

$\sharp 118$ : R. Agemi, H. Takamura, The lifespan of classical solutions to nonlinear wave equations in two space dimensions, 30 pages. 1991.

$\sharp 119$ : S. Altschuler, S. Angenent and Y. Giga, Generalized motion by mean curvature for surfaces of rotation, 15 pages. 1991.

$\sharp 120$ : T. Nakazi, Invariant subspaces in the bidisc and commutators, 20 pages. 1991.

$\sharp 121$ : A. Arai, Commutation properties of the partial isometries associated with anticommuting self-adjoint operators, 25 pages. 1991.

$\sharp 122$ : Y.-G. Chen, Blow-up solutions to a finite difference analogue of $u_{t}=\Delta u+u^{1+\alpha}$ in $N$-dimensional balls, 31 pages. 1991.

\#123: A. Arai, Fock-space representations of the relativistic supersymmetry algebra in the two-dimensional spacetime, 13 pages. 1991.

$\sharp 124$ : S. Izumiya, The theory of Legendrian unfoldings and first order differential equations, 16 pages. 1991.

$\$ 125$ : T. Hibi, Face number inequalities for matroid complexes and Cohen-Macaul ty types of Stanley-Reisner rings of distributive lattices, 17 pages. 1991.

\# 126: S. Izumiya, Completely integrable holonomic systems of first order differential equations, 35 pages. 1991.

$\sharp 127$ : G. Ishikawa, S. Izumiya and K. Watanabe, Vector fields near a generic submanifold, 9 pages. 1991.

$\sharp 128$ : A. Arai, I. Mitoma, Comparison and nuclearity of spaces of differential forms on topological vector spaces, 27 pages. 1991.

$\sharp 129$ : K. Kubota, Existence of a global solution to a semi-linear wave equation with initial data of non-compact support in low space dimensions, 53 pages. 1991.

$\sharp 130: \quad$ S. Altschuler, S. Angenent and Y. Giga, Mean curvature flow through singnlarities for surfaces of rotation, 62 pages. 1991.

$\sharp 131$ : M. Giga, Y. Giga and H. Sohr, $L^{p}$ estimates for the Stokes system, 13 pages. 1991 .

$\sharp 132$ : Y. Okabe, T. Ootsuka, Applications of the theory of $\mathrm{KM}_{2} \mathrm{O}$-Langevin equations to the non-linear prediction problem for the one-dimensional strictly stationary time series, 27 pages. 1992.

$\sharp 133$ : Y. Okabe, Applications of the theory of $\mathrm{KM}_{2} \mathrm{O}$-Langevin equations to the linear prediction problem for the multi-dimensional weakly stationary time series, 22 pages. 1992.

$\sharp 134$ : P. Aviles, Y. Giga and N. Komuro, Duality formulas and variational integrails, 22 pages. 1992.

$\sharp 135$ : S. Izumiya, The Clairaut type equation, 6 pages. 1992. 


\title{
SINGULAR SOLUTIONS OF FIRST ORDER DIFFERENTIAL EQUATIONS
}

\author{
S. IZUMIYA
}

Dedicated to the memory of Professor Giko Ikegami

\section{INTRODUCTION}

In classical treatises of equations (Carathéodory [2] , Courant-Hilbert [3], Forsyth [4] [5], Ince [8], Petrovski [14]) the discussions of equations with singular solutions are informal. In there, definitions of singular solutions are very confused. Even in modern articles ([9],[12]), it is studied under special assumptions. In this note we shall give a regorous definition of singular solutions of first order differential equations for real-valued functions (Theorem A).

On the other hand, the complete integrability is an important notion for the classical theory of first order differential equations. The notion of singular solutions has been usually appeared accompany with the notion of complete solutions in the above articles. Recently, we have studied some generic properties about completely integrable systems of first order differential equations as an application of the theory of Legendrian unfoldings ([7],[10],[11]). However, we have never seen a characterization of the complete integrability. Our another purpose is to give a characterization of complete integrability of first order differential equations (Theorem $\mathrm{B}$ ).

In $\S 1$, we shall state our main results. The proof of Theorem $A$ will be given in $\S 2$. We shall prove Theorem B in $\S 3$. Some typical examples will be given in $\S 4$.

All maps considered here are class $C^{\infty}$ unless stated otherwise.

ACKNowledgement. This work has been completed during the author's stay at the University of Liverpool mainly supported by Anglo-Japanese Scientific Exchange programme (The Royal Society and JSPS). The author would like to thank the Department of Pure Mathematics and especially Professor C.T.C. Wall for his kind encouragement and hospitality.

\section{MAIN RESULts}

In this section we shall state main results. An equation is a submersion germ $F$ : $\left(J^{1}\left(\mathbb{R}^{n}, \mathbb{R}\right),\left(x_{0}, y_{0}, p_{0}\right)\right) \rightarrow(\mathbb{R}, 0)$ on the 1 -jet space of functions of $n$-variables. Let $\theta$ be the canonical contact form on $J^{1}\left(\mathrm{R}^{n}, \mathrm{R}\right)$ which is given by $\theta=d y-\sum_{i=1}^{n} p_{i} d x_{i}$, where $(x, y, p)$ is the canonical coordinate of $J^{1}\left(\mathbb{R}^{n}, \mathbb{R}\right)$. We define a geometric solution of $F=0$ to be an immersion $i:\left(L, q_{0}\right) \rightarrow\left(J^{1}\left(\mathbb{R}^{n}, \mathbb{R}\right),\left(x_{0}, y_{0}, p_{0}\right)\right)$ of an $n$-dimensional manifold such that $i^{*} \theta=0$ and $i(L) \subset F^{-1}(0)$ (i.e. a Legendrian submanifold which is contained in $\left.F^{-1}(0)\right)$. We say that $\left(x_{0}, y_{0}, p_{0}\right)$ is a contact singular point if $\theta\left(T_{\left(x_{0}, y_{0}, p_{0}\right)} F^{-1}(0)\right)=0$. It is easy to show that $\left(x_{0}, y_{0}, p_{0}\right)$ is a contact singular point if and only if $F=F_{p_{i}}=$ $F_{x_{i}}+p_{i} F_{y}=0$ for $i=1, \ldots, n$ at $\left(x_{0}, y_{0}, p_{0}\right)$, where $F_{p_{i}}=\frac{\partial F}{\partial p_{i}}$ etc. We also say that $\left(x_{0}, y_{0}, p_{0}\right)$ is a $\pi$-singular point if $F=F_{p_{i}}=0$ for $i=1, \ldots, n$ at $\left(x_{0}, y_{0}, p_{0}\right)$. We denote the set of contact singular points by $\Sigma_{c}(F)$, the set of $\pi$-singular points by $\Sigma_{\pi}(F)$ and 
$\pi\left(\Sigma_{\pi}(F)\right)=D_{F}$, where $\pi(x, y, p)=(x, y)$. We call the set $D_{F}$ a discriminant set of the equation $F=0$. An equation $F=0$ is said to be completely integrable if there exists a foliation by geometric solutions on $F^{-1}(0)$. In this case such a foliation is called $a$ complete solution of $F=0$. Then we can define the notion of singular solutions. A geometric solution $i:\left(L, q_{0}\right) \rightarrow\left(J^{1}\left(\mathbb{R}^{n}, \mathbf{R}\right),\left(x_{0}, y_{0}, p_{0}\right)\right)$ of $F=0$ is called a singular solution of $F=0$ if it satisfies the following condition :

(*) For any representative $\tilde{i}: U \rightarrow F^{-1}(0)$ of $i$ and any open subset $V \subset U, \tilde{i}(V)$ is not contained in a leaf of any complete solutions of $F=0$.

Then we have the following theorem.

TheOREm A. For an equation $F:\left(J^{1}\left(\mathbf{R}^{n}, \mathbf{R}\right),\left(x_{0}, y_{0}, p_{0}\right)\right) \rightarrow(\mathbf{R}, 0)$ and a geometric solution $i:\left(L, q_{0}\right) \rightarrow\left(J^{1}\left(R^{n}, R\right),\left(x_{0}, y_{0}, p_{0}\right)\right)$ of $F=0$, the following conditions are equivalent.

(1) $i$ is a singular solution of $F=0$.

(2) There exists a complete solution of $F=0$ such that each leaves are transverse to $i$.

(3) Image $i \subseteq \Sigma_{c}(F)$.

We can also state another theorem as follows.

Theorem B. For an equation $F:\left(J^{1}\left(\mathbf{R}^{n}, \mathbf{R}\right),\left(x_{0}, y_{0}, p_{0}\right)\right) \rightarrow(\mathbf{R}, 0)$, the following are equivalent.

(1) $F=0$ is completely integrable.

(2) $\Sigma_{c}(F)=\emptyset$ or $\Sigma_{c}(F)$ is an $n$-dimensional submanifold.

Theorem $\mathrm{B}$ gives a characterization of complete integrability of equations. By the definition, if $\Sigma_{c}(F)$ is an $n$-dimensional submanifold, it is automatically a geometric solution of $F=0$. Then we have the following corollary of Theorems $\mathrm{A}$ and $\mathrm{B}$.

Corollary. An equation $F:\left(J^{1}\left(\mathrm{R}^{n}, \mathrm{R}\right),\left(x_{0}, y_{0}, p_{0}\right)\right) \rightarrow(\mathrm{R}, 0)$ has a singular solution if and only if $\Sigma_{c}(F)$ is an $n$-dimensional submanifold. Moreover, $\Sigma_{c}(F)$ is the singular solution of $F=0$.

\section{Proof of ThEOREM A}

In this section we shall give a proof of Theorem A. At first we give the proof that (1) implies (3).

Proof of Theorem A, (1) $\Rightarrow(3)$ : Let $i:\left(L, q_{0}\right) \rightarrow\left(J^{1}\left(\mathrm{R}^{n}, \mathrm{R}\right),\left(x_{0}, y_{0}, p_{0}\right)\right)$ be a singular solution of $F=0$. Suppose that there exists a point $q_{1} \in L$ such that $i\left(q_{1}\right)=\left(x_{1}, y_{1}, p_{1}\right) \notin$ $\Sigma_{c}(F)$. This means that $F=0$ is contact regular at $\left(x_{1}, y_{1}, p_{1}\right)$. By the classification theorem of the geometric theory of first order differential equations (see [13], Corollary 2 of Theorem 2.2.7), we may assume that $F(x, y, p)=p_{n}$. Then we now define a submanifold $E_{0}$ by $x_{n}=p_{n}=0$. It is easy to show that $E_{0}$ has a contact structure $\theta \mid E_{0}$. Since the contact Hamiltonian vector field of $p_{n}=0$ is given by $X_{p_{n}}=-\frac{\partial}{\partial x_{n}}$ (see [13], Theorem 1.4.3) and $i$ is a solution of $p_{n}=0$, then $-\frac{\partial}{\partial x_{n}} \in T i(L)$. It follows that $\ell_{0}=i(L) \cap\left\{x_{n}=0\right\}$ is an $(n-1)$-dimensional submanifold in $E_{0}$. Since $i$ is a Legendrian immersion, then $\ell_{0}$ is also a Legendrian submanifold of $E_{0}$. By the Darboux's theorem, we can easily show that there exists a foliation on $E_{0}$ with leaves are Legendrian submanifold of $E_{0}$ such that $\ell_{0}$ is a leaf of this foliation. Since $-\frac{\partial}{\partial x_{n}} \notin T E_{0}$, then each leaves are isotropic submanifold of $J^{1}\left(\mathbb{R}^{n}, \mathbb{R}\right)$ with the non-characteristic property. Thus we can solve the "parametrized" 
Cauchy problem by ordinary characteristic method (see [13] Proposition 1.5.3), then we have a local complete solution of $p_{n}=0$ such that $i(L)$ is a leaf of this solution. This contradicts to the definition of the singular solution.

We can also prove that (2) implies (1).

Proof of Theorem A, (2) $\Rightarrow(1)$ : Let $\tilde{i}: U \rightarrow F^{-1}(0)$ be a representative of $i$. If there exist an open subset $V \subset U$ and a complete solution such that $\tilde{i}(V)$ is contained in a leaf of such a foliation. Then there exists transversal foliations on $F^{-1}(0)$ around $\tilde{i}(V)$ whose leaves are Legendrian submanifolds. Then $F^{-1}(0)$ is an isotropic submanifold around $\tilde{i}(V)$. This contradicts to the fact that dimensions of isotropic submanifolds are at most $n$.

We need some preparations to prove that (3) implies (2).

Let $F:\left(J^{1}\left(\mathbb{R}^{n}, \mathbf{R}\right),\left(x_{0}, y_{0}, p_{0}\right)\right) \rightarrow(\mathbb{R}, 0)$ be an equation such that $\left(x_{0}, y_{0}, p_{0}\right)$ is a contact singualr point. If $F_{y}=0$ at $\left(x_{0}, y_{0}, p_{0}\right)$, then $F_{x_{i}}=F_{p_{i}}=0$ at $\left(x_{0}, y_{0}, p_{0}\right)$ for any $i=$ $1, \ldots, n$. This contradicts to the fact that $F$ is a submersion germ. Then we have $F_{y} \neq 0$. By the implicit function theorem, there exists a function germ $h:\left(T^{*} \mathbb{R}^{n},\left(x_{0}, p_{0}\right)\right) \rightarrow \mathbb{R}$ such that $F^{-1}(0)=\{(x, y, p) \mid y=h(x, p)\}$, where $T^{*} \mathbb{R}^{n}$ is the cotangent bundle of $\mathbb{R}^{n}$. We may consider that $J^{1}\left(\mathbb{R}^{n}, \mathbb{R}\right) \cong T^{*} \mathbb{R}^{n} \times \mathbb{R}$. In the terminology of Kossowski [12] the equation of the above form is called a graphlike equation. We now define a map germ

$$
\operatorname{graph}(h):\left(T^{*} \mathbb{R}^{n},\left(x_{0}, p_{0}\right)\right) \rightarrow\left(J^{1}\left(\mathbf{R}^{n}, \mathbf{R}\right),\left(x_{0}, y_{0}, p_{0}\right)\right)
$$

by

$$
\operatorname{graph}(h)(x, p)=(x, h(x, p), p) .
$$

We set a 1 -form on $T^{*} R^{n}$ by $\theta_{h}=\operatorname{graph}(h)^{*} \theta=d h-\sum_{i=1}^{n} p_{i} d x_{i}$. Then we have the following one to one correspondence.

$$
\begin{gathered}
\{L \mid L \text { is a solution of } y-h(x, p)=0\} \\
\operatorname{graph}(h) \uparrow \downarrow \Pi_{*}
\end{gathered}
$$

$\left\{L \mid i: L \subset T^{*} \mathbb{R}^{n}\right.$ is a maximal integral submanifold of $\left.\theta_{h}=0\right\}$,

where $\Pi(x, y, p)=(x, p)$ and $\Pi_{*}(L)=\Pi(L)$. By this reason, a solution of graphlike equation $y-h(x, p)=0$ may be regarded as a maximal isotropic submanifold of $\left(T^{*} \mathrm{R}^{n}, \theta_{h}\right)$. Since $-d \theta_{h}=\sum_{i=1}^{n} d p_{i} \wedge d x_{i}$ is the canonical symplectic two form, then a solution of $y-h(x, p)=0$ is a Lagrangian submanifold of $\left(T^{*} \mathrm{R}^{n}, \omega\right)$, where $\omega=-d \theta_{h}$. For the definition and properties of Lagrangian submanifolds, see [1]. We now refer the following very important result.

THEOREM 2.1. (Kostant-Sternberg [6]) Let $(P, \omega)$ be a symplectic manifold, $L$ a Lagrangian submanifold and $\alpha$ a smooth 1 -form on $P$ with $\alpha \mid L=0$ and $d \alpha=\omega$. Then there exists a tubular neighbourhood $V$ of $L$ in $P$, and a unique vector bundle isomorphism $K: V \rightarrow\left(T^{*} L, \theta_{L}\right)$ such that $K$ is the identity on $L$ and $K^{*} \theta_{L}=\alpha$. Here, $\theta_{L}$ is the canonical 1-form on $T^{*} L$.

Now we can prove that (3) implies (2). 
Proof of THEOREM A, (3) $\Rightarrow(2)$ : Since Image $i \subseteq \Sigma_{c}(F)$, we may assume that $F=$ $y-h(x, p)$. In our case, graph $(h)^{-1} \circ i(L)=L_{h}$ is a Lagrangian submanifold of $T^{*} \mathrm{R}^{n}$. We may apply the Kostant-Sternberg theorem to conclude that there exists a tubular neighbourhood $V$ of $L_{h}$ in $T^{*} R^{n}$ and a unique vector bundle isomorphism $K: V \rightarrow$ $\left(T^{*} L_{h}, \theta_{L_{h}}\right)$ such that $K$ is the identity on $L_{h}$ and $K^{*} \theta_{L_{h}}=-\theta_{h}$. Since the fibre of the cotangent bundle $T^{*} L_{h} \rightarrow L_{h}$ are maximal integral submanifolds of $\theta_{L_{h}}=0$, these fibres make a foliation whose leaves are solution of $F=0$ and tansverse to $i(L)$. This completes the proof.

\section{Proof of Theorem B}

In order to prove Theorem $\mathrm{B}$, we need some preparations. We say that an $n$-parameter family of function germs $f:\left(\mathbb{R}^{n} \times \mathbb{R}^{n},\left(t_{0}, x_{0}\right)\right) \rightarrow\left(\mathrm{R}, y_{0}\right)$ is a classical complete solution of $F=0$ if $F\left(x, f(t, x), f_{x}(t, x)\right)=0$ and $\operatorname{rank}\left(f_{t_{i}}, f_{t_{i} x_{j}}\right)=n$. Then we define a map germ $j_{*}^{1} f:\left(\mathbf{R}^{n} \times \mathrm{R}^{n},\left(t_{0}, x_{0}\right)\right) \rightarrow\left(J^{1}\left(\mathrm{R}^{n}, \mathrm{R}\right),\left(x_{0}, y_{0}, p_{0}\right)\right)$ by $j_{*}^{1} f(t, x)=\left(x, f(t, x), f_{x}(t, x)\right)$. Since $f(t, x)$ is a classical complete solution, then $j_{*}^{1} f$ is a local parameterization of $F^{-1}(0)$. It follows that the family $\left\{\text { Image } j_{*}^{1} f_{t}\right\}_{t \in\left(\mathbb{R}^{n}, t_{0}\right)}$ gives a local foliation on $F^{-1}(0)$. For a Legendrian immersion germ $i:\left(L, q_{0}\right) \rightarrow J^{1}\left(\mathbb{R}^{n}, \mathbf{R}\right), q_{0} \in L$ is said to be a Legendrian singular point if $\pi \circ i$ is not an immersion at $q_{0}$. We remark that $q_{0}$ is a Legendrian nonsingular point if and only if $\tilde{\pi} \circ i$ is a local diffeomorphism at $q_{0}$, where $\tilde{\pi}(x, y, p)=x$. Then we have the following lemma.

LEMMA 3.1. 1) If $F=0$ has a classical complete solution, then $\Sigma_{c}(F)=\emptyset$ or $\Sigma_{c}(F)$ is an $n$-dimensional submanifold.

2) If each leaves of a complete solution of $F=0$ are Legendrian non-singular, then it is a classical complete solution.

Proof: 1) By the above argument, $j_{*}^{1} f$ is a local parameterization of $F^{-1}(0)$. Since $j_{*}^{1} f^{*} \theta=\sum_{i=1}^{n} f_{t_{i}}(t, x) d t_{i}$, then $j_{*}^{1} f(t, x) \in \Sigma_{c}(F)$ if and only if $f_{t_{i}}(t, x)=0$ for $i=$ $1, \ldots, n$. Since rank $\left(0, f_{t_{i} x_{j}}\right)=\operatorname{rank}\left(f_{t_{i}}, f_{t_{i} x_{j}}\right)=n$ at $j_{*}^{1} f(t, x) \in \Sigma_{c}(F)$, then we have $\operatorname{rank}\left(f_{t_{i} x_{j}}, f_{x_{i} x_{j}}\right)=n$. It follows that $\Sigma_{c}(F)$ is an $n$-dimensional submanifold.

2) Suppose that there exists a complete solution with leaves are Legendrian non-singular. Then we have an $n$-parameter family of smooth sections

$$
s:\left(\mathbf{R}^{n} \times \mathbf{R}^{n},\left(t_{0}, x_{0}\right)\right) \rightarrow\left(J^{1}\left(\mathbf{R}^{n}, \mathbf{R}\right),\left(x_{0}, y_{0}, p_{0}\right)\right)
$$

of $\tilde{\pi}$ (i.e. $\tilde{\pi} \circ s(t, x)=x$ ) such that $s$ is an immersion, $s\left(\mathbb{R}^{n} \times \mathbb{R}^{n}\right)=F^{-1}(0)$ and $s_{t}^{*} \theta=0$ for any $t \in\left(\mathbb{R}^{n}, t_{0}\right)$, where $s_{t}(x)=s(t, x)$. It follows that there exists a family of function germs $f:\left(\mathbb{R}^{n} \times \mathbb{R}^{n},\left(t_{0}, x_{0}\right)\right) \rightarrow\left(\mathbb{R}^{n}, y_{0}\right)$ such that $j_{*}^{1} f(t, x)=s(t, x)$. Since $s$ is an immersion, then $f$ is a (classical) complete solution of $F=0$.

Now we can prove Theorem $B$.

Proof of TheOREM B: If $\Sigma_{c}(F)=\emptyset$, then $F=0$ is contact regular at $\left(x_{0}, y_{0}, p_{0}\right)$. It follows that the classical existence theorem (see [13]) that there exists a complete solution of $F=0$. If $\Sigma_{c}(F)$ is an $n$-dimensional submanifold, then it is also a solution of $F=0$. In this case it has been already proved in the proof of Theorem $\mathrm{A}((3) \Rightarrow(2))$.

Suppose that there exists a complete solution of $F=0$. Let $\left(L,\left(x_{0}, y_{0}, p_{0}\right)\right)$ be a germ of a leaf of the complete solution at the point $\left(x_{0}, y_{0}, p_{0}\right)$. By Arnol'd-Zakalyukin's theory 
([1], Corollary 20.2), there exist a partition $(I, J)$ of the set $\{1, \ldots, n\}$ and a function germ $S\left(x_{I}, p_{J}\right)$ such that

$$
L=\left\{\left(x_{I},-\frac{\partial S}{\partial p_{J}}, S\left(x_{I}, p_{J}\right)-<\frac{\partial S}{\partial p_{J}}, p_{J}>, \frac{\partial S}{\partial x_{I}}, p_{J}\right)\right\}
$$

where $\left\langle x_{J}, p_{J}\right\rangle$ is the canonical inner product. We now define a contact diffeomorphism germ by

$$
C_{(I, J)}(x, y, p)=\left(x_{I}, p_{J}, y-<x_{J}, p_{J}>, p_{I},-x_{J}\right) .
$$

Then we have $C_{(I, J)}(L)=\left\{\left(x_{I}, p_{J}, S\left(x_{I}, p_{J}\right), \frac{\partial S}{\partial x_{I}}, \frac{\partial S}{\partial p_{J}}\right)\right.$. It follows that $C_{(I, J)}(L)$ is Legendrian non-singular at $C_{(I, J)}\left(\left(x_{0}, y_{0}, p_{0}\right)\right)$. By Lemma 3.1 , we have a classical complete solution on $F \circ C_{(I, J)}^{-1}=0$. Then $\Sigma_{c}(F)=C_{(I, J)}^{-1}\left(\Sigma_{c}\left(F \circ C_{(I, J)}^{-1}\right)\right)$ is also an $n$-dimensional submanifold. This complete the proof.

\section{EXAMPLES}

In this section we shall give typical examples of equations with singular solution.

Examples 4.1, The Clatraut equation. The following is the classical example of an equation with singular solution : $y=\sum_{i=1}^{n} x_{i} p_{i}+f\left(p_{1}, \ldots, p_{n}\right)$, where $f$ is a smooth function. The complete solution is given by $y=\sum_{i=1}^{n} x_{i} t_{i}+f\left(t_{1}, \ldots, t_{n}\right)$ and the singular solution is the envelope of graphs of complete solution.

Example 4.2, The Dual of The Clairaut equation. Consider the equation : $y=$ $f\left(x_{1}, \ldots, x_{n}\right)$. This equation is given by the Legendre transform (see [9]) of the Clairaut equation. The complete solution is given by $\left\{(t, f(t), u) \mid(t, u) \in \mathbb{R}^{n} \times \mathbb{R}^{n}\right\}$, where $t=$ $\left(t_{1}, \ldots, t_{n}\right)$ is the parameter.

The singular solution is given by $\Sigma_{c}(F)=\left\{\left(x, f(x), f_{x}(x)\right) \mid x \in \mathbf{R}^{n}\right\}$. We can observe that $F^{-1}(0)=\Sigma_{\pi}(F) \supset \Sigma_{c}(F)$.

EXAMPLE 4.3. Consider the following equation : $y-2 p^{3}=0$. We can show that $\Sigma_{\pi}(F)=$ $\Sigma_{c}(F)=\{(x, 0,0) \mid x \in \mathbb{R}\}$ which is a singular solution. We also have a complete solution $s:(\mathbb{R} \times \mathbf{R}, 0) \rightarrow J^{1}(\mathbb{R}, \mathbb{R})$ given by $s(u, t)=\left(3 u^{2}+t, 2 u^{3}, u\right)$, where $t$ is the parameter. In this case the singular solution is a locus of cusps of the complete solution (not an envelope!).

\section{REFERENCES}

1. V.I. Arnol'd, S.M. Gusein-Zade and A.N. Varchenko, "Singularities of differentiable maps, vol. 1, Monographs in Math. 82," Birkhauser, 1985.

2. C. Carathéodory, "Calculus of Variations and Partial Differential Equations of First Order, Part I, Partial Differential Equations of the First Order," Holden-Day, 1965.

3. R.Courant and D. Hilbert, "Methods of mathematical physics I, II," Wiley, 1962.

4. A. R. Forsyth, "Theory of differential equations, Part $I I$ partial differential equations," Cambridge Univ. Press, London, 1906.

5. A. R. Forsyth, "A Treatise on differential equations," Macmillan and Co, 1885.

6. M. Golubitsky and V. Guillemin, Contact equivalence for Lagrange manifolds, Adv. In Math. 15 (1975), 375-387.

7. A. Hayakawa, G. Ishikawa, S. Izumiya and K. Yamaguchi, Classification of generic integral diagram and first order ordinary differential equations, preprint. 
8. E. L. Ince, "Ordinary differential equations," Dover, 1926.

9. S. Izumiya, Generic properties of first order partial differential equations, Proceedings of Topology conference in Hawaii (Birkhäuser) (1991) (to appear).

10. S. Izumiya, The theory of Legendrian unfoldings and first order differential equations, preprint.

11. S. Izumiya, Classification of generic completely integrable holonomic systems of first order differential equations, preprint.

12. M. Kossowski, First order partial differential equations with singular solution, Indiana Univ. Math. Jour. 35 (1986), 209-223.

13. V. V. Lychagin, Local classification of non-linear first order partial differential equations, Russian Math. Surveys 30 (1975), 105-175.

Department of Pure Mathematics, University of Liverpool, P.O.Box 147, Liverpool L69 3BX, England UK and Department of Mathematics, Faculty of Science, Hokkaido University, Sapporo 060 JAPAN 